\title{
ORIGINAL
}

\section{FACTORES ASOCIADOS AL CONTROL DE LA HIPERTENSION ARTERIAL EN PERSONAS MAYORES DE 60 AÑOS EN ESPAÑA}

\author{
Rafael Tuesca-Molina (1), Pilar Guallar-Castillón (2), José Ramón Banegas-Banegas (2) y Auxilia- \\ dora Graciani-Pérez Regadera (2)
}

(1) Universidad del Norte, Barranquilla, Colombia. Departamento de Salud Familiar y Comunitaria. División Ciencias de la Salud.

(2) Universidad Autónoma de Madrid. España. Departamento de Medicina Preventiva, Salud Pública y Microbiología. Facultad de Medicina.

\section{RESUMEN}

Fundamento: En la última década se ha observado una mejora en el control de la hipertensión arterial en España. Dicho control repercute en la disminución de la morbimortalidad cardiovascular. El objetivo de este trabajo es identificar los factores asociados al control de la hipertensión arterial en varones y mujeres hipertensos diagnosticados que reciben tratamiento farmacológico.

Métodos: Estudio descriptivo transversal, realizado a partir de los datos del estudio: «Hipertensión arterial y otros factores de riesgo en la población de 60 años y más de España». La muestra, incluyó a 1.461 hipertensos diagnosticados tratados farmacológicamente, seleccionados por muestreo probabilístico y polietápico. La información se obtuvo por entrevista en el domicilio sobre el control de la hipertensión arterial, variables sociodemográficas, estilo de vida, uso de servicios de salud y calidad de vida relacionada con la salud.

Resultados: No se observaron diferencias de genero en el control de la hipertensión $(\mathrm{p}=0,09)$. El control en varones se relaciona con residir en área rural $(\mathrm{OR}=1,83$; IC al 95\%: 1,06-3,14); ser soltero $(\mathrm{OR}=3,40$; IC al $95 \%: 1,32-8,74)$ y realizar actividad física (OR=1,69; IC al 95\%:1,06-2,69). Las mujeres que consumen alcohol de forma moderada controlan mejor la hipertensión $(\mathrm{OR}=1,63$; IC al $95 \% 1,14-2,33)$

Conclusiones: Esta investigación determina según el género algunos factores relacionados con el control de la hipertensión en hipertensos diagnosticados y tratados farmacológicamente. En varones, el control se asoció significativamente con: residir en área rural, ser soltero y realizar alguna actividad física. Mientras, que en las mujeres el control se asoció con consumir alcohol de forma moderada.

Palabras clave: Hipertensión arterial. España. Enfermedades cardiovsculares.

Correspondencia:

Rafael Tuesca-Molina

Departamento de Salud Familiar y Comunitaria.

Universidad del Norte.

Kilómetro 5 Antigua carretera a Puerto Colombia.

Correo electrónico: rtuesca@uninorte.edu.co
ABSTRACT

\section{Factors Associated the Control of Hypertension among Older Spaniards, over 60 Years of Age}

Background: In the last decade, improvement has been observed in the control of arterial hypertension in Spain. Such control has an effect in the decrease of cardiovascular morbidity and mortality. The objective of the study was to identify factors associated to the control of arterial hypertension in awareness of hypertension among males and females who receive pharmacological treatment.

Methods: A cross-sectional analysis, made in basis of the following study data: «Arterial Hypertension and other risk factors in the population of 60 years old and more in Spain». The sample included 1461 hypertensive patients treated pharmacologically, selected by probabilistic and multistage sampling. The information recollected about the control of arterial hypertension, social and demographic variables, lifestyle, healthcare service usage, and life quality related to health, by residence interviewing.

Results: No differences between gender were observed in the control of hypertension $(\mathrm{p}=0,09)$, In men control were significantly linked to: residence in rural areas $(\mathrm{OR}=1,83$; $\mathrm{CI}$ at $95 \%$ : 1,06-3,14); being single $(\mathrm{OR}=3,40$; $\mathrm{CI}$ at $95 \%$ : 1,32-8,74); and exercising $(\mathrm{OR}=1,69 ; \mathrm{CI}$ at $95 \%: 1,06-2,69)$. Women who consume alcohol in a moderate way controlled themselves more $(\mathrm{OR}=1,63$; CI at $95 \%$ $1,14-2,33)$

Conclusions: This research determines, according to gender, some factors related with the control of arterial hypertension in awareness of hypertension patients treated pharmacologically. In male patients the control is related to: living in rural areas, being single and physical activity. While in females control was associated with moderate alcohol consumption.

Key words: Hypertension. Cardiovascular diseases. Spain. 


\section{INTRODUCCIÓN}

La hipertensión arterial (HTA), es un factor de riesgo cardiovascular de tipo causal muy prevalente en nuestro medio, con una elevada y conocida morbimortalidad que se incrementa cuando se añaden otros factores de riesgo cardiovascular como son: el tabaquismo, la hipercolesterolemia y la hiperglucemia $^{1,2}$.

Diversos estudios epidemiológicos han descrito repetidamente un incremento del riesgo de enfermedad cardiovascular a medida que aumentan los niveles de presión arterial sistólica y diastólica. Dichos estudios muestran una correlación positiva, continua e independiente entre las cifras de presión arterial y la incidencia de eventos coronarios $^{1,3-4}$.

La necesidad de una mayor reducción de las cifras de presión arterial en la población hipertensa está bien establecida por diversos ensayos clínicos. De igual manera diversos protocolos de actuación y guías de práctica clínica elaborados por diferentes organismos y sociedades científicas ${ }^{3,5-8}$ reiteran la necesidad de mejorar y lograr el control adecuado de la hipertensión arterial, lo que se constituye en si mismo como la meta a lograr.

La falta de control de la presión arterial y de otros factores de riesgo cardiovascular contribuyen a un incremento de complicaciones cardiovasculares que se constituye en la primera causa de muerte de los pacientes que la padecen y genera más de 5 millones de estancias hospitalarias en el territorio español ${ }^{9-11}$.

En España el progreso en el control de la hipertensión ha sido aproximadamente de un $1 \%$ anual en los estudios en población atendida en Atención Primaria ${ }^{2}$. La tasa de control en el país muestra cifras entre el $13 \%$ y menos del $40 \%$, muy similar a otros países desarrollados. Con respecto a estas cifras el margen de variación obedece a factores como: 1) el punto de corte para establecer la cifra de control de hipertensión en personas mayores (160/96 mmHg frente a 140/90); 2) El tipo de muestra empleada en los estudios (pequeñas y no representativas); 3) El ámbito del estudio (población general, atención primaria, unidades de hipertensos etc.); 4) Áreas geográficas con distinta prevalencia; 5) Metodología heterogénea en la medición de presión arterial y, finalmente, la descripción de tasas de control en relación con el tipo de hipertensos (tratados, diagnosticados con tratamiento o al total de hipertensos).

El presente trabajo tiene como objetivo determinar los factores asociados con el control de la hipertensión arterial en hombres y mujeres hipertensos diagnosticados y tratados con fármacos $\mathrm{y}$, de acuerdo con variables de tipo sociodemográficas, su relación con el uso de servicios sanitarios, los estilos de vida y la calidad de vida relacionada con la salud.

\section{SUJETOS Y MÉTODOS}

El estudio se realizó con los datos del estudio: «Hipertensión arterial y otros factores de riesgo en la población de 60 años y más de España», referenciado en otros estudios previamente publicados, en el que participaron 4.009 sujetos con representatividad estadística a nivel nacional, y que se seleccionaron mediante muestreo probabilístico por conglomerados y polietápico. Estudios previos detectaron que el $65 \%$ de los sujetos hipertensos eran hipertensos diagnostica$\operatorname{dos}^{12,13}$. La muestra estuvo constituida por 1.461 personas hipertensas diagnosticadas que manifestaron recibir tratamiento farmacológico. Se les realizó una encuesta que recogió información sobre el control de su hipertensión arterial y las variables: sociodemográficas, estilo de vida, uso de servicios de salud y calidad de vida relacionada con la salud, seguida de un examen físico en el que se midió la presión arterial y las variables antropométricas. En todos los casos se obtu- 
vo consentimiento informado de los participantes del estudio o de familiares.

La presión arterial se midió con esfigmomanómetros de mercurio calibrado. En cada individuo se realizaron en condiciones estandarizadas 6 tomas de la presión arterial, a lo largo de dos ocasiones separadas (tres mediciones en cada ocasión). Se consideró a un individuo hipertenso cuando su presión arterial sistólica (PAS) era $\geq$ de 140 mm de Hg. o su presión arterial diastólica (PAD) era $\geq$ de $90 \mathrm{~mm}$ de $\mathrm{Hg}$. o recibía tratamiento farmacológico para controlar su presión arterial ${ }^{3-8}$.

Se consideró a un sujeto como hipertenso diagnosticado y tratado farmacológicamente, cuando a la pregunta: ¿Le indicó (el médico) un tratamiento con pastillas? respondía «Sí, y lo hago en la actualidad». Se estimaron hipertensos controlados a quienes presentaron niveles de presión arterial sistólica (PAS) $<140 \mathrm{~mm} \mathrm{Hg}$. y su presión arteri-

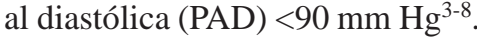

La información en el domicilio de las personas participantes la recogió un equipo entrenado en el Departamento de Salud Pública de la universidad autónoma de Madrid. La investigación tuvo el respaldo del Comité ético del Hospital universitario La Paz, de Madrid. Para el estudio de las variables independientes se adaptaron cuestionarios de las Encuestas Nacionales de Salud de España ${ }^{14}$, el «Estudio de los estilos de vida de la población adulta española» ${ }^{15} \mathrm{y}$ el cuestionario SF36 de calidad de vida relacionada con la salud traducido al español $(\mathrm{CVRS})^{16}$.

Dentro de las variables independientes las sociodemográficas fueron clasificadas de la siguiente manera: la edad se determinó en años cumplidos; para su presentación fue reagrupada en grupos decenales en tres intervalos: 60 a 69 años, 70 a 79 años y el último grupo edad superior a 80 años.
A fin de valorar el lugar de residencia, se midió el hábitat más allá de la dicotomía urbano-rural ${ }^{17}$ para controlar la medición del fenómeno en estudio; para ello se introdujo una tercera categoría quedando los criterios de clasificación del hábitat medidos como: rural, urbano y litoral (Cantábrico y Mediterráneo). Esta selección se apoyó con: el plano cartográfico y de carreteras $1 / 1000000$ $1 \mathrm{~cm}$ de Michelín ${ }^{\circledR}{ }^{18}$. Se asignó el área rural a aquellos sujetos que residían en municipios con menos de 5.000 habitantes, exceptuando las áreas que estuviesen próximas al litoral. El área urbana fue asignada a municipios por encima de 10.000 habitantes y se exceptúan áreas que estuviesen con proximidad al litoral. Para definir litoral se estimó aquellos asentamientos con proximidad al litoral Cantábrico y/o Mediterráneo, independiente del número de habitantes.

Para clasificar el ejercicio físico en tiempo libre se preguntó a los sujetos de estudio: ¿Qué tipo de ejercicio físico realizan durante su tiempo libre?. Las opciones de respuesta se desglosan en cuatro categorías: a) No hago ejercicio; b) Alguna actividad física o deportiva ocasional; c) Actividad física regular, varias veces al mes; d) Entrenamiento físico varias veces a la semana. Para la presentación de resultados esta variable fue agrupada de manera dicotómica: sí realiza actividad física (quien seleccionó cualquier ítem diferente de a.) y no efectúa actividad física o sedentario quien respondió la opción a.

La CVRS se midió con la versión española del cuestionario SF- $36^{16}$. que consta de 36 ítems que valoran 8 componentes o escalas relacionados con: función física, rol físico, dolor corporal, salud general, vitalidad, función social, rol emocional y salud mental. Además se siguieron métodos estandarizados y se calculó el índice sumarial del componente físico y mental.

En el presente estudio se utilizaron únicamente las sub-escalas de la función social, la 
salud general y los índices sumarios del componente físico y mental.

Para medir el cumplimiento terapéutico se utilizó el test de Morisky-Green ${ }^{19}$, compuesto por 4 preguntas diseñadas de forma sencilla y clara que sirven para detectar el cumplimiento o adherencia al tratamiento farmacológico en los hipertensos.

Otras variables incluidas fueron: convivencia del sujeto, consumo de tabaco, índice de masa corporal (IMC), número de enfermedades crónicas, visita médica al domicilio y asistencia a consulta médica.

El análisis estadístico se realizó con el paquete informático SPSS para Windows versión 11.5 en español, para varones y mujeres. Para estimar el efecto independiente de cada variable explicativa se usó la odds ratios y su respectivo intervalo de confianza al $95 \%$ y se construyeron modelos de regresión logística múltiple no condicional en variables que alcanzaron un valor de $p<0,05$ en el análisis bivariado. Además, se mantuvo la edad en los modelos según grupos decenales. Se calculó la p de tendencia lineal, introduciendo las variables como continuas en los modelos. Las variables que aparecen en la tabla 1 provienen del análisis de la $\chi^{2}$ al comparar los grupos de hipertensos diagnosticados y no diagnosticados con el resto de variables categóricas. Se usó el test de la t de Student para comparar esta variable con las variables continúas; seguido de ello, las variables que obtuvieron un valor «p» inferior a 0,20 se analizaron de manera bivariada.

\section{RESULTADOS}

La tasa de respuesta para varones y mujeres fue del 95,9\%. El 65\% de los sujetos conocían su diagnóstico de hipertensión. Dentro de este grupo el $85 \%$ de los hipertensos diagnosticados estaban tratados con fármacos y el $29,6 \%$ estaban controlados (figura 1).

Figura 1

Flujograma de los sujetos hipertensos conocidos según su tratamiento y control
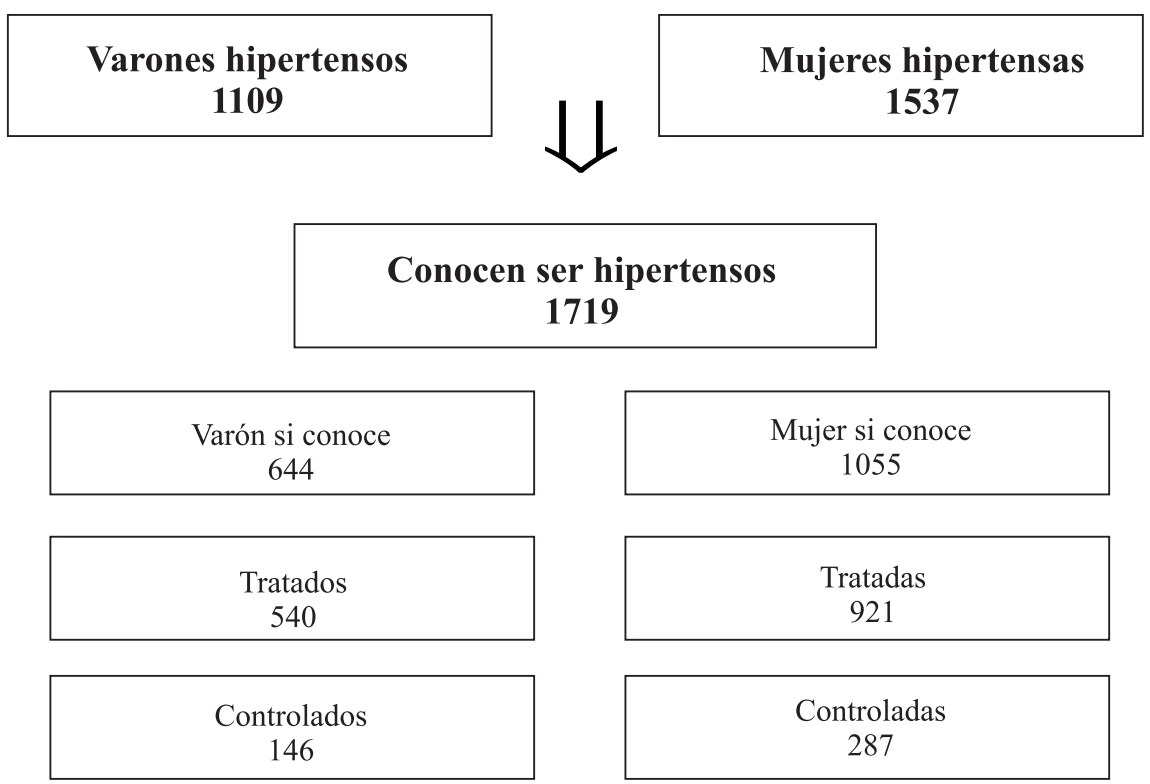


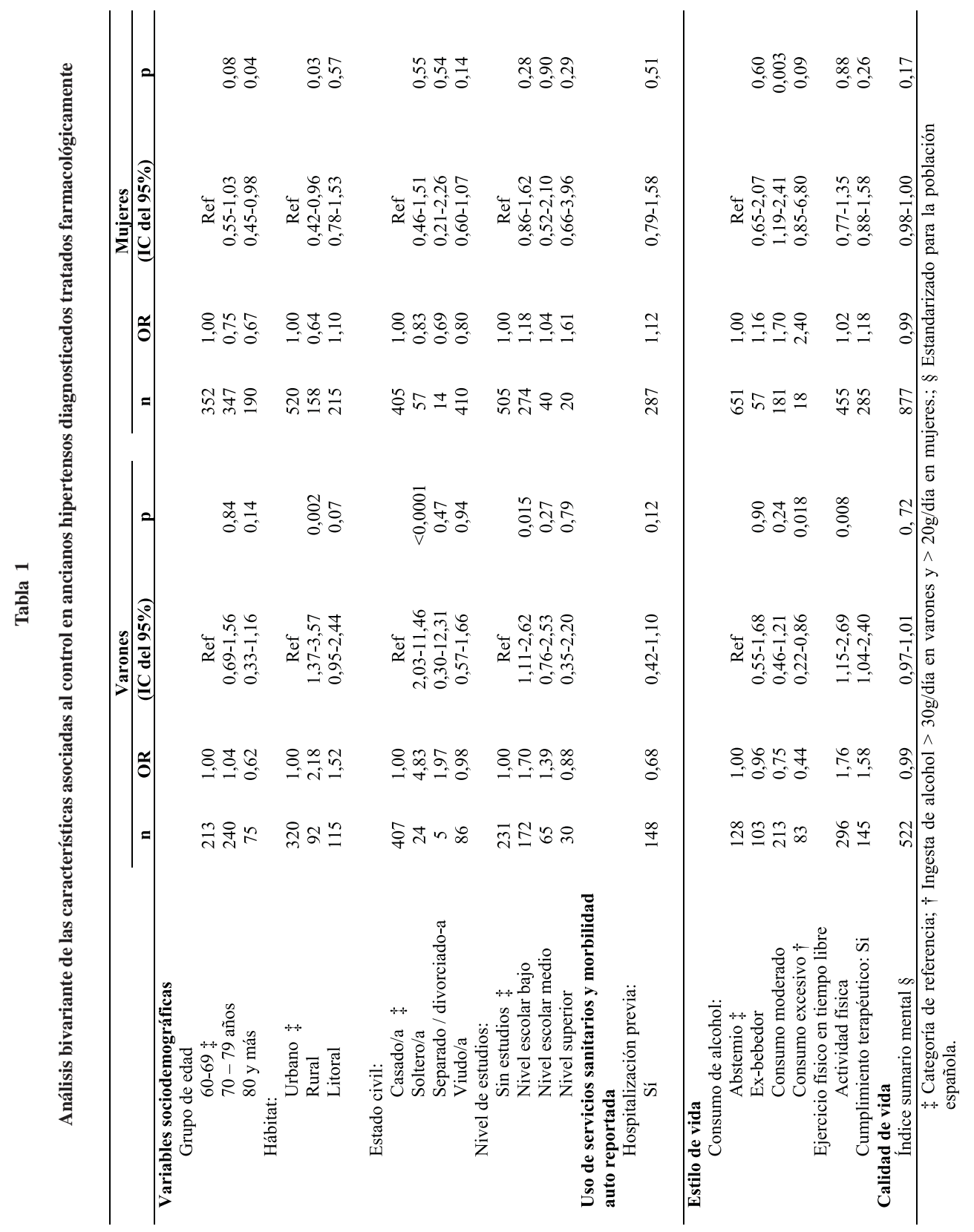


Tabla 2

Análisis multivariante de las características asociadas al control de la hipertensión en varones hipertensos diagnosticados tratados farmacológicamente

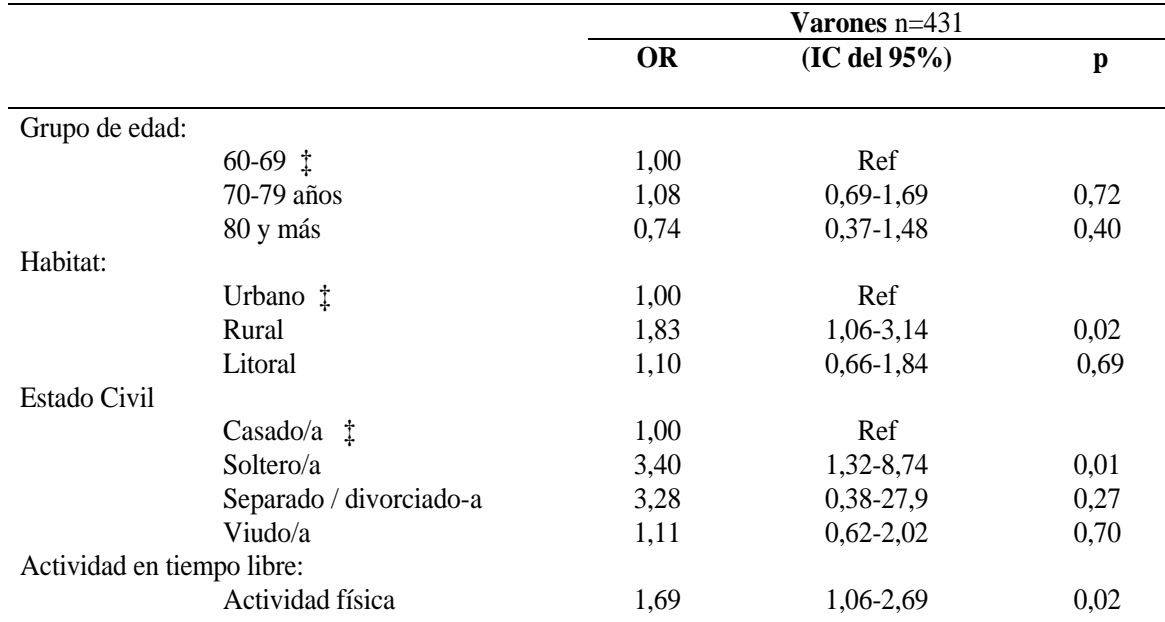

‡ Categoría de referencia; Ingesta de alcohol > 30g/día en varones y > 20g/día en mujeres. Ajustado por edad, hábitat, estado civil, nivel de estudios, actividad física en tiempo libre, cumplimiento terapéutico y consumo de alcohol.

Tabla 3

Análisis multivariante de las características asociadas al control de la hipertensión en mujeres hipertensas conocidas tratadas farmacológicamente

\begin{tabular}{cccc}
\hline & \multicolumn{3}{c}{ Mujeres n=885 } \\
\cline { 2 - 4 } & OR & (IC del 95\%) & p \\
\hline Grupo de edad: & & \\
60-69 $\ddagger$ & 1,00 & Ref & \\
$70-79$ años & 0,76 & $0,55-1,05$ & 0,10 \\
80 y más & 0,72 & $0,48-1,06$ & 0,10 \\
Abstemio $\ddagger$ & 1,00 & & \\
Ex-bebedor & 1,16 & $0,65-2,09$ & 0,60 \\
Consumo moderado & 1,63 & $1,14-2,33$ & 0,007 \\
Consumo excesivo $\dagger$ & 2,07 & $0,72-5,91$ & 0,17
\end{tabular}

Categoría de referencia

$\dagger$ Ingesta de alcohol > 30g/día en varones y > 20g/día en mujeres.

Ajustado por edad, hábitat, consumo de alcohol. 
El porcentaje de los sujetos hipertensos tratados y controlados de este grupo fue del $27,0 \%$ en varones y del $31,1 \%$ en mujeres, sin encontrarse diferencias significativas $(\mathrm{p}=0,09)$. (figura 1$)$.

Con relación a los factores asociados al control de la hipertensión arterial en varones (tabla 1) tuvieron mayor control: los solteros (OR=4,83; IC al 95\%:2,03-11,46); los que refieren tener bajo nivel de escolaridad $(\mathrm{OR}=1,70$; IC al 95\%:1,11-2,62), los que manifiestan realizar actividad física en tiempo libre $(\mathrm{OR}=1,76$; IC al 95\%:1,15-2,69) y además presentan disminución en la presión de pulso ( $\mathrm{OR}=0,89$; IC al 95\%:0,88-0,91). Mientras que el escaso control en varones se relaciona con el consumo excesivo de alcohol (OR=0,44; IC al 95\%: 0,22-0,86)

Al considerar los factores asociados al nocontrol de la hipertensión arterial en mujeres (tabla 1) se encontró que las mayores de 80 años se controlan menos $(\mathrm{OR}=0,67$; $\mathrm{IC}$ al 95\%:0,45-0,98), lo mismo que las que residen en área rural $(\mathrm{OR}=0,64$, IC al 95\%:0,42$0,96)$. Quienes consumen alcohol de forma moderada se controlan más $(\mathrm{OR}=1,70$; $\mathrm{IC}$ al $95 \% 1,19-2,41$ ), además se observó una disminución en la presión de pulso $(\mathrm{OR}=0,88$; IC al 95\%:0,87-0,99).

En el análisis multivariado en los varones hipertensos diagnosticados y tratados (tabla 2) se observó que el control de la hipertensión estaba asociado con residir en el área rural $(\mathrm{OR}=1,83$; IC al 95\%: 1,06-3,14), ser soltero $(\mathrm{OR}=3,40 ; \mathrm{IC}$ al $95 \%: 1,32-8,74)$ y realizar actividad física en tiempo libre (OR=1,69; IC al 95\%:1,06-2,69). En las mujeres (tabla 3) el control de la hipertensión se asocia con el consumo de alcohol de forma moderada $(\mathrm{OR}=1,63$; IC al 95\% 1,14-2,33).

\section{DISCUSIÓN}

De este estudio destacan de los aspectos novedosos y relacionados con el control de la hipertensión en sujetos mayores de 60 años. En primer lugar, si bien es cierto que las cifras de control procedente de diversos estudios no permiten su comparación de manera directa, se observa cierta tendencia en el incremento del grado de control de la presión arterial. Sin intentar extrapolar el resultado de la tasa de control en personas mayores obtenido en nuestro estudio para varones y mujeres $(27,03 \%$ vrs $31,16 \%)$, los hallazgos se ubican en puntos intermedios con respecto de la variación del grado de control en el estudio de CONTROLPRES realizado durante el periodo de 2001 $(28,8 \%)^{20}$ al $2003(38,8 \%)^{10}$. Con respecto a los hallazgos relacionados con la prevalencia del conocimiento, tratamiento y control de la hipertensión en esta población, se observa cierto grado de similitud con las tendencias reportadas en NHANES en los Estados Unidos para el periodo 1999-2000 ${ }^{1,11} \mathrm{y}$ otros países desarrollados ${ }^{20-23}$.

Con respecto al género no se encontraron diferencias estadísticamente significativas en el control de la presión arterial $(\mathrm{p}=0,09)$, sin embargo cabe señalar que publicaciones previas consideran que las mujeres conocen más que son hipertensas, se tratan más y se controlan mejor ${ }^{1,4,21-25}$. Las razones presumibles para explicar nuestro hallazgo muy probablemente estén relacionadas con las tendencias positivas en el tratamiento y control de la hipertensión en el territorio español. Cabe resaltar que se deben valorar algunos factores tales como: el seguimiento en el consejo medico, el nivel de educación o conocimiento con respecto a la enfermedad y el tiempo bajo terapia según las directrices internacionales.

Otro aspecto de interés corresponde al hábitat. En este trabajo se muestran diferencias con relación al control en varones y mujeres. Los varones en el área rural se controlan más, mientras que las mujeres se controlan menos. Higgs ${ }^{17}$ plantea que existen marcadas diferencias entre el hábitat urbano y rural, inclinándose de manera positiva al sector rural (mejores condiciones y estilos 
de vida). Otros autores plantean que la prevalencia y el control de la hipertensión se consideran como buenos predictores de las variaciones geográficas de la mortalidad cerebrovascular. A este respecto, consideramos válido incluir una tercera categoría en nuestro trabajo que corresponde al litoral. Esta asignación guarda relación con el posible efecto espacial de asentamientos a fin de explicar variaciones geográficas en la morbilidad y como determinante de salud, especialmente en el control, no abordado en investigaciones previas a nivel nacional.

Un aspecto importante a valorar concierne al hecho de tener nivel de estudios bajo y relacionarse con mejor control en los varones. Diversas investigaciones argumentan lo contrario a nuestro hallazgo, es decir, que la baja escolaridad en varones está relacionada con un pobre control ${ }^{21-29}$. Frente a este hallazgo es posible que se identifique si el médico asume un rol activo en educar al paciente de manera que explique esta diferencia. Si bien es cierto, la mujer hipertensa conoce más su situación hipertensiva que el varón $^{13,20-24}$ este hallazgo suscita pensar que la posible explicación estaría relacionada además de lo anterior en que los varones mayores presentan mayor adherencia al tratamiento que las mujeres y presumiblemente que no todas las mujeres participen por igual en el cuidado ${ }^{30}$. En este mismo sentido nos resulta sorprendente que los varones solteros se controlen más que quienes tienen pareja, si se asume que el papel de la pareja o de la familia se considera como factor protector de la salud y fuente de apoyo social que refuerza la adopción de conductas saludables ${ }^{31}$.

Con respecto a las bondades de realizar actividad física se destaca su rol para disminuir la presión arterial sistólica y diastóli$\mathrm{ca}^{32}$. Por lo tanto, esta recomendación se debe reforzar en las mujeres y estimular a los pacientes hipertensos a incrementar esta actividad saludable junto con otras medidas no farmacológicas.
Un aspecto que diferencia este estudio de otros es el número de mediciones empleado para estimar la presión arterial. La mayoría de los estudios establecen una o dos tomas, mientras que en este se realizaron 6 tomas de la presión, lo que permite una mejor estimación. El promedio de PAS en varones y mujeres controlados en este trabajo fue de $126,50 \mathrm{mmHg} \pm 11,02$ y $128,51 \mathrm{mmHg} \pm$ 9,60 respectivamente y la PAD de 74,48 \pm 8,56 en varones y $74,94 \mathrm{mmHg} \pm 8,31$ en mujeres, lo que muestra un mejor control. A este respecto, el objeto del control está en aunar esfuerzos para lograr cifras por debajo de 140/90 u óptimas, las cuales reducen la aparición de complicaciones ${ }^{3,10,27}$.

En nuestro estudio el control de la hipertensión en las mujeres está relacionado con el consumo moderado de alcohol y quienes consumen de forma excesiva (los varones) controlan menos. Este hecho guarda semejanza con los hallazgos de Nieto en población americana ${ }^{33}$.

En resumen, estos argumentos tienen implicaciones prácticas en la atención del anciano, lo cual equivale a valorar el rol del consejo medico y redireccionar las estrategias preventivas, a fin de lograr un reconocimiento de la situación de esta problemática, más si se tienen en cuenta las bondades y ventajas del tratamiento farmacológico y no farmacológico frente a las complicaciones ${ }^{34}$. De forma adicional a esta consideración, es importante zanjear las discrepancias y establecer alianzas con los sanitarios locales e intervenir los factores de riesgos relacionados en esta investigación con el control de la hipertensión y así mismo fortalecer los factores protectores.

En este sentido, la tarea y el reto consiste por un lado en continuar la investigación en líneas no estudiadas hasta el momento ${ }^{35}$ : efecto de la terapia no farmacológica en el control, valorar el impacto de diferentes estrategias en el mejoramiento del cumplimiento terapéutico. evaluar los procesos de 
calidad en la atención del hipertenso y aplicar de manera racional las guías internacionales para lograr un manejo uniforme de la hipertensión arterial ${ }^{3,5-8}$ a fin de reducir las desigualdades regionales, haciendo énfasis en grupos de riesgo que apunte a mejorar el control de la hipertensión que, a la postre, conllevaría un incremento de los niveles de conocimiento, tratamiento y control.

\section{AGRADECIMIENTOS}

Este trabajo se ha financiado con ayudas parciales de investigación de la Universidad Autónoma de Madrid. Además, Rafael Tuesca Molina ha disfrutado de una beca doctoral de la Fundación Universidad del Norte durante la realización de este trabajo. Los organismos financiadores no han tenido participación alguna en la recogida, interpretación de los datos, ni en la decisión de enviar el manuscrito para publicación.

\section{BIBLIOGRAFÍA}

1. Hajjar I, Kotchen T. Trends in prevalence, awareness, treatment, and control of hypertension in the United States, 1988-2000. JAMA 2003;290(2): 199-206.

2. Villar-Álvarez F, Banegas, J.R., Donado-Campos J.d.M y Rodríguez-Artalejo, F. Las Enfermedades Cardiovasculares y sus Factores de Riesgo en España: hechos y cifras. Informe SEA 2003. Madrid: ERGON; 2003

3. Chobanian AV, Bakris GL, Black HR, Cushman WC, Green LA, Izzo JL, Jr. et al. The Seventh Report of the Joint National Committee on Prevention, Detection, Evaluation, and Treatment of High Blood Pressure: the JNC 7 report. JAMA 2003;289:2560-72.

4. Lloyd-Jones D, Evans J, Larson M, O`Donnell C, Roccella E, Levy D. Differential control of systolic and diastolic blood pressure. Factors associated with lack of blood pressure control in the community. Hypertension 2000;36:594-99.

5. Laurent S. Guidelines from the British Hypertension Society. BMJ 2004;328:593-4. 2003 European
Society of Hypertension-European Society of Cardiology guidelines for the management of arterial hypertension. J Hypertens. 2003;21:1011-53.

6. Brotons C, Royo-Bordonada MA, Alvarez-Sala L, Armario P, Artigao R, Conthe P, et al. Adaptación española de la Guía Europea de Prevención Cardivascular. Rev. Esp. Salud Pública. 2004;78(4):435438.

7. Williams B, Poulter NR, Brown MJ, Davis M, McInnes GT, Potter JF et al. British Hypertension Society guidelines for hypertension management 2004 (BHS-IV): summary. BMJ 2004;328:634-40.

8. Banegas JR, Rodriguez Artalejo F, de la Cruz JJ, de Andrés B, Rey J.Mortalidad relacionada con la hipertensión y la presión arterial en España. Med Clin.1999;112:489-94.

9. Coca A, Evolución del control de la hipertensión arterial en atención primaria en España. Resultados del estudio Controlpres 2003. Hipertensión.2005;22(1)5-14.

10. Andros V. Uncontrolled blood pressure in a treated, high-risk managed care population. Am J Manag Care. 2005;11(7):S215-S219.

11. Banegas JR, Rodríguez-Artalejo F, Ruilope LM, Graciani A, Luque M, de la Cruz-Troca JJ et al. Hypertension magnitude and management in the elderly population of Spain. J.Hypertens. 2002; 20(11):2157-64.

12. Tuesca-Molina R, Guallar-Castillón P, BanegasBanegas J.R., Graciani-Pérez A Factores asociados con el conocimiento de la hipertensión en españoles mayores de 60 años. Hipertensión. En prensa 2006.

13. Ministerio de Sanidad y Consumo. Encuesta Nacional de Salud de España 1997. Madrid: Ministerio de Sanidad y Consumo, 1999.

14. Gil-López E, Jiménez GP R, Pérez-Andrés C, Vargas-Marcos F. Estudio de los estilos de vida de la población adulta española. Madrid: Artegraf, S.A.ed; 1992.

15. Unitat de Recerca en Serveis Sanitaris. Manual de puntuación de la versión Española del cuestionario de Salud SF-36. Barcelona: Institut Municipal d’Investigacio Medica.; 1998

16. Higgs G. Investigating trends in rural health outcomes: a research agenda. Geoforum.1999;30:203221. 
17. España - Portugal. 21(990). [mapa de carreteras] Poitiers - France, Michelin Ediciones. 2000 Morisky DE, Green LW, Levine DM. Concurrent and predictive validity of a self-reported measure of medication adherence. Med Care.1986;24:67-74.

18. Coca A. Evolución del control de la hipertensión arterial en España. Resultados del estudio Controlpres 2001. Hipertensión. 2002;19(9):390-99.

19. Steckelings UM, Stoppelhaar M, Sharma AM, Wittchen H-U, Krause P, Kupper B, et al. HYDRA: Possible determinants of unsatisfactory hypertension control in German primary care patients.Blood Pressure.2004;13:80-88

20. Trenkwalder P, Ruland D, Stender M, Gebhard J, Trenkwalder C, Lydtin H et al. Prevalence, awareness, treatment and control of hypertension in a population over the age of 65 years: results from the Starnberg Study on Epidemiology of Parkinsonism and Hypertension in the Elderly (STEPHY). J.Hypertens. 1994;12:709-16.

21. van Rossum CT, van de MH, Witteman JC, Hofman A, Mackenbach JP, Grobbee DE. Prevalence, treatment, and control of hypertension by sociodemographic factors among the Dutch elderly. Hypertension 2000;35:814-21.

22. Menotti A, Lanti M, Zanchetti A, Puddu PE, Cirillo M, Mancini $\mathrm{M}$ et al. Impact of the Gubbio population study on community control of blood pressure and hypertension. Gubbio Study Research Group. J.Hypertens. 2001;19:843-50.

23. De Backer G, Myny K, De Henauw S, Doyen Z, Van Oyen H, Tafforeau J et al. Prevalence, awareness, treatment and control of arterial hypertension in an elderly population in Belgium. J.Hum.Hypertens. 1998;12:701-6.

24. Nieto FJ, Alonso J, Chambless LE, Zhong M, Ceraso M, Romm FJ et al. Population awareness and control of hypertension and hypercholesterolemia. The Atherosclerosis Risk in Communities study. Arch.Intern.Med. 1995;155:677-84.
25. McNagny SE, Ahluwalia JS, Clark WS, Resnicow KA. Cigarette smoking and severe uncontrolled hypertension in inner-city African Americans. Am.J.Med 1997;103:121-7.

26. Woo J, Ho S, Yuen YK, Chan S, Yu A, Lau J. Prevalence and effectiveness of treatment of hypertension on cardiovascular morbidity and mortality in an elderly population aged 70 years and over. Arch.Gerontol.Geriatr 1997;25:159-65.

26. Rooks R, Simonsick E, Miles T, Newman A, Kritchevsky $\mathrm{S}, \mathrm{Schulz} \mathrm{R}$ et al. The association of race and socioeconomic status with cardiovascular disease indicators among older adults in the health, aging, and body composition study. J Geron 2002;57B:S247-S256.

27. García-Calvente M, Mateo-Rodríguez I, Eguiguren A. El sistema informal de cuidados en clave de desigualdad. Gac Sanit. 2004; 18 (Sup 1):132-9.

28. Rohlfs I, Borrell C, Anitua C, Artazcoz L, Colomer C, Escribá V. et al. La importancia de la perspectiva de género en las encuestas de salud. Gac Sanit. 2000;14(2):146-55

29. Brandao Rondon MU, Alves MJ, Braga AM, Teixeira OT, Barretto AC, Krieger EM et al. Postexercise blood pressure reduction in elderly hypertensive patients. J.Am.Coll.Cardiol. 2002;39:676-82.

30. Nieto FJ, Alonso J, Chambless LE, Zhong M, Ceraso M, Romm FJ et al. Population awareness and control of hypertension and hypercholesterolemia. The Atherosclerosis Risk in Communities study. Arch.Intern.Med. 1995;155:677-84.

31. Nickey W, Lenfant C, Chobanian AV, Roccella EJ. The National High Blood Pressure Education Program: Longtime Partners With New Strategies. JAOA 2003;103:297-9.

32. Banegas JR. Epidemiología de la hipertensión arterial en España. Situación actual y perspectivas. Hipertensión.2005;22 (9):353-62. 